\title{
Placenta Accreta at 15 Weeks of Gestational Age and Uterus Preservation with Hysterectomy Abortion
}

\author{
Farahnaz Farzaneh,, ${ }^{1,}$ and Arezoo Esmailzadeh ${ }^{2}$ \\ ${ }^{1}$. Infertility Fellowship, Gynecologist, Infectious Disease and Tropical Medicine Research Center of Zahedan University of Medical Sciences, Zahedan, Iran \\ ${ }^{2}$ Assistant Professor, Department of Obstetrics and Gynecology, Zahedan University of Medical Sciences, Zahedan, Iran \\ "Corresponding author: Farahnaz Farzaneh, Infertility Fellowship, Gynecologist, Infectious Disease and Tropical Medicine Research Center of Zahedan University of Medical \\ Sciences, Zahedan, Iran. Tel: +98-9144263014, E-mail: farahnaz1826@yahoo.com
}

Received 2017 February 03; Revised 2017 June 12; Accepted 2017 October 24.

\begin{abstract}
Placenta accrete refers to an abnormality of placental implantation in which the anchoring placental villi attached to myometrium rather than decidua resulting in a morbidity adherent placenta. Placenta increta (chorionic villi penetrate into the myometrium) and placenta percreta (chorionic villi penetrate through the myometrium to the uterine serosa or adjacent organs) are related, but more severe, abnormalities of placental implantation. The pathogenesis is primarily attributed to defective decasualization of the implantation site. Placenta accreta has been recognized mostly in the third trimester however may also present in second trimester. It has very heavy, life-threatening hemorrhage to both the mother and fetus. The authors' report a patient with two previous cesarean deliveries, who had been referred for pregnancy termination from Iranian legal medicine organization with 15 weeks of gestational age and placenta acctera. In this patient, uterus preservation and hysterectomy abortion was performed. The case totally lost $1500 \mathrm{cc}$ blood and she was discharged from hospital two days later, with feeling of well- being. We have examined this patient after 1.5 months, she wasn't vaginal bleeding and the uterus was in the pelvic and serum BHCG (with titer) was negative.
\end{abstract}

Keywords: Placenta Accreta, Hysterotomy Abortion, Uterus Preservation

\section{Introduction}

Abnormal placenta invasion during pregnancy is known Accreta, increta, and percreta based on invasion of placental villi in to the uterine wall [1]. Placenta Accreta is much more common than placenta increta and precreta. Placenta Accreta is abnormality of placental villis attach to myometrium rather than decidua, resulting in a morbidity adherent placenta. Based on two studies, the type and frequency of abnormal placentation were: placenta acreta: 79 percent, planenta increta: 14 percent, placenta percreta: 7 percent. In 1950 Placenta acreta was rare occurring 1 in 30000 deliveries in the USA. During the 1980s and 1990s the incidence increased.

Pathogenesis of placenta accrete is not known with certainly, the most common theory is that defective decidualization related to previous surgery or to anatomical factors (endocervix, lower uterine segment, endosalpinx, uterine anatomy). This is supported by the associated with 80 percent of these cases. Individuals with risk factors for placenta accreta or percreta should be diagnosed during last of first trimester or early second trimester pregnancy. In the patients with history of previous cesarean section or curettages, the physician could use conservative techniques like uterine artery embolization (UAE) or methotrexate injection prior to curettage [2].

\section{Case Presentation}

We present 32 years old patient with two previous cesarean deliveries, who was referred for pregnancy termination with 15 weeks of gestation age and placenta acctera from Iranian legal medicinal organization. She presented normal vital sign and normal lab analysis $(\mathrm{HB}=$ 11.9, $\mathrm{HCT}=26.1, \mathrm{PLT}=292000, \mathrm{WBC}=9400, \mathrm{BS}=80, \mathrm{Cr}$ $=0.5, \mathrm{PT}=13, \mathrm{PTT}=23$ ). After preparation of the patient and blood products, the patient has transferred to operation room and was underwent general anesthesia and low midline abdomen incision, after opening the viseral peritoneum, the author observed placenta with only visceral peritoneum and pulled out male dead fetus and placenta, there was relatively sever bleeding from the placental sites, then the author trim the incision and did figure of 8 sutures to placenta sites and placed one intra uterus $24 \mathrm{~F}$ balloons with $30 \mathrm{cc}$ instilled water and bleeding was controlled, The uterus layers with vicry 1 in 2 layers have been closed. Hemovac drain was prepared on the incision and closed the abdomen layers. Through surgery the patient was transfused with three units of isogroup crossmached packed cell and three units of FFP. After surgery the patient has been transferred to ICU and one day later hemovac drain was removed and the patient was discharged 2 days later. Patient lost $1500 \mathrm{cc}$ blood through the surgery, and 
underwent a complicated surgery. Prevalence of placenta accrete and increta in early pregnancy has been increased due to prominent rising in cesarean section, curettage and endometritis. This research presents approach with placenta accreta in 15th of gestational age and compares it with other similar cases from another article.

\section{Discussion}

Placenta accrete shows abnormality of placenta invasion in which placental villi was attached into the myometrium without intervening decidua. The pathogenesis of placenta accreta is not known for the us. The most common cause is that lacking of decidualization (thin, poorly formed, or absent decida) to previous surgery. 80 percent of these cases have a history of previous cesarean section, curretage or myomectomy (A. Wen 1999, I.E. TimorTritsch 2012) (2.4). Risk factors of placenta percreta include prior cesarean section or other uterine surgery, Multiparity, prior curettage, placenta previa, or prior manual removal of placenta, endometrial infection causing trauma to endometrium. The most important risk factor of placenta accrete is placenta previa after a prior cesarean delivery. There are a few patients with placenta percreta in early pregnancy in zahedan and other cities because of difficulty diagnosis of these cases in the early periods of pregnany but placenta accrete as compared to percreta is common.

Our case had a history of two prior low segment transverse cesarean sections (LSCS). And she was stable for pregnancy termination that we did laparotomy and hysterectomy with uterus preservation. Another case presented at 17 weeks of gestation age with an acute abdomen and an rising of alpha feto protein (A. Esmans 2004) [1] and Two other cases presented at 18 weeks and 21 weeks gestation, (Rashbaum W. K. 1995, Komiya K. 2009) [3]. These patients had no risk factors other than a single episode of pelvic inflammatory disease [4]. While rupture of uterus in placenta accreta during early pregnancy is happened, hysterectomy because of hemoperitoneum and hypovolemic shock is necessary. In Japan, Turkey, Mexico, and Germany have reported a few cases with uterine rupture in placenta accrete [5-9]. All these patients underwent hysterectomy. This complication occurs due to thinning of the myometrium.

Ideally, placenta accrete is first suspected because of finding on obstetrical ultrasound examination while the patient is asymptomatic. The abnormally implanted placenta is often diagnosed on prenatal sonographic evaluation of the placenta in a woman with risk factors for Accrete (previa, previous caesarian), but may be an accidental finding.
The first clinical manifestation of placenta accrete is usually profuse, life-threading hemorrhage that occurs at the time of attempted manual placental separation. Part or all of the placenta remains strongly attached to the uterine cavity, and no plane of separation can be developed. However it also may present as antenatal bleeding in the setting of placenta previa.

In the era of increased cesarean deliveries, we should be able to diagnosis of increta and accreta, to prevent maternal mortality and morbidity.

Several studies have reported an association between placenta accrete and otherwise unexplained elevation in second trimester maternal serum alpha fetoprotein (MSAFP) concentration ( $>2$ or $2.5 \mathrm{MOM}$ ). Although an elevated MSAFP level supports an ultrasound-based diagnosis of placenta accrete, but a normal AFP does not exclude the diagnosis.

Imaging tools in the evaluation of abnormal placentation include-Doppler Sonography, gray-scale Sonography, and MRI [10]. Sonogrphy can detect loss of placental homogeneity, which is replaced by multiple intraplacental sonolucent spaces adjacent to the involved myometrium and loss of irregularity of the normal hypoechoic area behind the placenta and Retroplacental myometrial thinning (thickness of $<1 \mathrm{~mm}$ ) and color Doppler could be diagnosis diffuse or focal intraparenchymal lacunar flow, vascular lakes with turbulent flow, hypervascularity of serosa-bladder interface, prominent subplacental venous complex. MRI had high diagnostic accuracy for detection of placenta accrete (sensivity $94.4 \%$ and specifity $84 \%$ ). Accuracy of diagnosis with MRI is highly dependent on the expertise and experience of the radiologist interpreting the image. Uterine bulging into bladder, Heterogeneous signal intensity within the placenta, Presence of intraplacental bands on the T2W imaging, abnormal placental vascularity and Focal interruption of the myometrium are MRI findings. MRI may be useful than ultrasound in two clinical scenarios: 1: evaluation of a possible posterior placenta accrete because the bladder cannot be used to help clarity the placental myometrial interface, and 2: assessment of the depth of myometrial and parametrial involvement and if the placenta is anterior, bladder involvement.

When a placenta accrete or incerta is diagnosed early in the pregnancy with the use of ultrasonography, MRI, and color Doppler, hysterectomy will be the most option surgery, but conservative methods like uterine artery embolization (UAE) and hysteroscopy with lesion resection, mostly in combination could be performed [11]. Some researchers have used methotrexate, in combination with curettage or embolization. Conservative treatment options although help to preserve the uterus, are not without complications. 


\subsection{Conclusion}

Placenta accreta is dangerous obstetric complication with maternal mortality and morbidity. It is rarely seen in first trimester of pregnancy. Women with a placenta previa or a low anterior placenta and prior uterine surgery should have through Sonographic evaluation of the interface between the placenta and myometrium between about 18 and 24 weeks of gestation. At this gestational age, the diagnosis is suspected. Color flow Doppler can help support a Sonographic diagnosis of placenta accrete. MRI may be useful when the ultrasound finding are uncertain, If placenta accrete or worse is suspected the patient and her family should be counseled, about the suspected placental abnormality and an appropriate delivery plan can be developed [10]. Even Hysterectomy seemed to be the only adequate treatment available but we can try to do conservative surgeries (UAE, MTX + hysteroscopy) for these patients

\section{Acknowledgments}

All authors had equal role in design, work, statistical analysis and manuscript writing.

\section{Footnotes}

Conflict of Interests: The authors declare no conflict of interest.

Funding/Support: Zahedan University of Medical Sciences.

\section{References}

1. Esmans A, Gerris J, Corthout E, Verdonk P, Declercq S. Placenta percreta causing rupture of an unscarred uterus at the end of the first trimester of pregnancy: case report. Hum Reprod. 2004;19(10):24013. doi: 10.1093/humrep/deh421. [PubMed: 15298972].

2. Wen A, Qian D, Zhang X. [Placenta accreta in early pregnancy: a case report and review of the literature]. Zhonghua Fu Chan Ke Za Zhi. 1999;34(10):606-8. [PubMed: 11477800].

3. Rashbaum WK, Gates EJ, Jones J, Goldman B, Morris A, Lyman WD. Placenta accreta encountered during dilation and evacuation in the second trimester. Obstet Gynecol. 1995;85(5 Pt 1):701-3. doi: 10.1016/00297844(95)00050-2. [PubMed: 7724099].

4. Komiya K, Saitou K, Inoue S, Igarashi T, Hirabayashi Y, Seo N. [Massive hemorrhage associated with undiagnosed placenta percreta in a second-trimester pregnancy receiving abortion procedure]. Masui. 2009;58(8):1036-8. [PubMed: 19702228].

5. Fleisch MC, Lux J, Schoppe M, Grieshaber K, Hampl M. Placenta percreta leading to spontaneous complete uterine rupture in the second trimester. Example of a fatal complication of abnormal placentation following uterine scarring. Gynecol Obstet Invest. 2008;65(2):81-3. doi: 10.1159/000108288. [PubMed: 17851255].

6. Moriya M, Kusaka H, Shimizu K, Toyoda N. Spontaneous rupture of the uterus caused by placenta percreta at 28 weeks of gestation: a case report. J Obstet Gynaecol Res. 1998;24(3):211-4. doi: 10.1111/j.14470756.1998.tb00077.x. [PubMed: 9714992].

7. Kinoshita T, Ogawa K, Yasumizu T, Kato J. Spontaneous rupture of the uterus due to placenta percreta at 25-weeks' gestation: a case report. J Obstet Gynaecol Res. 1996;22(2):125-8. doi: 10.1111/j.14470756.1996.tbo0953.x. [PubMed: 8697340].

8. Topuz S. Spontaneous uterine rupture at an unusual site due to placenta percreta in a 21-week twin pregnancy with previous cesarean section. Clin Exp Obstet Gynecol. 2004;31(3):239-41. [PubMed: 15491074].

9. Bernal-Martinez S, Chavez H, Villa F, Guzman A. [Uterine rupture and placenta percreta in the second trimester. Presentation of a case]. Ginecol Obstet Mex. 1996;64:482-3. [PubMed: 8974956].

10. Available from: https://www.uptodate.com.

11. Yu M, Liu XY, Dai Q, Cui QC, Jin ZY, Lang JH. [Diagnosis and treatment of placenta accreta in the second trimester of pregnancy]. Zhongguo Yi Xue Ke Xue Yuan Xue Bao. 2010;32(5):501-4. doi: 10.3881/j.issn.1000503X.2010.05.006. [PubMed: 21050552]. 